\title{
Minilaparotomy Cholecystectomy: A Forgotten Art in Laparoscopic Cholecystectomy Era (Our Experience from a Peripheral Hospital in North India)
}

\author{
Bharath N Kumar ${ }^{1}$ and Rahul Pandey (iD) ${ }^{2, *}$ \\ ${ }^{1}$ Command Hospital (Southern Command), Pune, India \\ ${ }^{2}$ Department of Surgery, 155 Base Hospital, Tezpur, India \\ "Corresponding author: Department of Surgery, 155 Base Hospital, Tezpur, India. Email: rahuladviksimpy@gmail.com
}

Received 2021 May 16; Accepted 2021 October 25.

\begin{abstract}
Background: This study aimed to report the experience of performing minilaparotomy cholecystectomy in a peripheral hospital by a single surgeon.

Methods: Data collected from 50 consecutive patients undergoing minilaparotomy cholecystectomy by a single surgeon over 18 months at a peripheral hospital were reviewed and studied. The recorded data encompassed demographics, operating time, incision size, conversion rate to open cholecystectomy, perioperative complications, and hospital stay duration.

Results: Fifty consecutive patients, who underwent minilaparotomy cholecystectomy for symptomatic cholelithiasis, were studied, among whom 48 patients were females. The participants' mean age was 45 years. The length of the surgical incision was $4.5-6 \mathrm{~cm}$, and only three patients required conversion to open cholecystectomy. The average operating time was 60 minutes; and the average postoperative hospital stay was 2.14 days.

Conclusions: Minilaparotomy cholecystectomy is comparable with laparoscopic cholecystectomy in terms of postoperative morbidity, and it is ideal for peripheral hospitals lacking laparoscopic facilities.
\end{abstract}

Keywords: Minilaparotomy Cholecystectomy, Laparoscopic Cholecystectomy, Minimally Invasive Surgery

\section{Background}

Following the introduction of laparoscopic cholecystectomy (LC) in the late 1980s, symptomatic cholelithiasis treatment underwent a rapid revolution. It became an acceptable alternative due to its various advantages such as smoother postoperative course, less pain, early return to work, and better cosmesis compared to conventional open cholecystectomy. Minilaparotomy cholecystectomy (MC) was first introduced in 1982 to reduce morbidity, limit the post-operative scar and pain associated with the conventional open cholecystectomy (1). Although LC is currently considered the gold standard, some surgeons consider mini-laparotomy as an alternative to LC, especially in centers where laparoscopic facilities are not available since it needs no special training and equipment and is also cost effective (2).

Most of the peripheral hospitals in developing nations are still not equipped with laparoscopic facilities, and MC may turn out to be a viable alternative in such hospitals.

\section{Methods}

This retrospective study was carried out at a peripheral hospital in India during 18 months, from July 2010 to Dec 2011. Fifty consecutive patients with symptomatic cholelithiasis and chronic cholecystitis, were included in this study, while their age, gender, or co-morbidities did not matter. The pre-operative evaluation of the patients comprised a detailed history, including a review of previous medical and surgical records, meticulous physical examination, laboratory tests, and an ultrasound scan of the abdomen.

MC was performed under either general anesthesia with or without epidural analgesia or combined spinal anesthesia and epidural analgesia (CSE) according to the anesthesiologist's preference. All patients were given a preoperative dose of antibiotic (Inj Cefotaxime $1 \mathrm{gm}$ ) at the 
anesthesia induction time, followed by three postoperative doses at eight-hour intervals as antibiotic prophylaxis.

A $4.5-6 \mathrm{~cm}$ incision was given, starting just short of midline and running obliquely parallel to $3 \mathrm{~cm}$ below the right costal margin (Figure 1). The anterior rectus sheath was divided in the direction of the incision. The rectus muscle was either cut with diathermy or split along its fibers and retracted medially. The posterior sheath, transversalis fascia, and peritoneum were divided in the incision direction. The gall bladder (GB) was removed by either the retrograde or 'cystic duct-first' technique or the antegrade or 'fundus-first' technique, based upon the operative findings, namely the Calot's triangle anatomy and adhesions. The cystic duct and artery were ligated with 2/0 silk sutures. No specialized retractors or headlights were used during the surgery (Figure 2). The abdominal wall was closed with a non-absorbable polypropylene suture, and the skin was closed with a skin stapler or subcuticular monofilament absorbable suture. Intravenous fluids were discontinued after 24 hours.

The patients were followed up three months after the operation. The data recorded included demographics, operating time, incision size, operative findings, conversion rate to open cholecystectomy, perioperative complications, postoperative analgesic requirement, and hospital stay duration. The term 'operating time' was defined as the period from the skin incision to skin closure. Descriptive analyses were then performed.

\section{Results}

During the study period, fifty patients referring to this center with symptomatic chronic calculous cholecystitis were included, among whom 48 patients were females, and only two patients were males. The patients' mean age was 45 years (range 25 - 70 years), and the mean body mass index (BMI) was $29.3 \mathrm{Kg} / \mathrm{sq} . \mathrm{mt}$. Only five patients had comorbidities; two had diabetes mellitus, two had hypertension, and one had diabetes and hypertension. MC was performed through a 4.5 - $6 \mathrm{~cm}$ incision (Figure 3). Cholecystectomy was possible through minilaparotomy in 47 patients (94\%). IN this regard, only three patients (6\%) needed the incision to be extended. The rectus muscle was split in 13 cases, and it was cut with diathermy in 37 cases. GB was removed using the 'cystic duct-first' approach in 18 patients and the 'fundus-first' approach in 32 patients. The average operating time was 60 minutes (40 - 150 minutes).
The drain was placed in only one male patient having a severely contracted GB with extensive adhesions and difficult dissection. The average postoperative hospital stay was 2.14 days (range 2 - 5 days). The analgesic requirement in the postoperative period was 3-5 parenteral doses. Then all patients required only oral analgesics. The average time to return to routine work was nine days (7 - 12 days). There was an intra-operative complication in only one patient, who had a small contracted GB and extensive adhesions between GB, transverse colon, small bowel, and omentum. The small bowel was inadvertently injured during the dissection of the adhesions; however, it was identified to be immediately and primarily sutured, and the wound was closed with no drain placement. The same patient developed wound seroma in the postoperative period and later superficial secondary infection, which required debridement and secondary suturing under local anesthesia. None of the other 49 patients had any complication and there was no mortality in the study.

\section{Discussion}

The present era of keyhole surgery was pioneered by the advent of LC in 1985 (3). Although LC is widely accepted as a treatment alternative for symptomatic cholelithiasis (4). MC has the advantage of minimal invasiveness similar to LC; however, it is less expensive and requires any no specialized training. This study aimed to analyze the MC outcomes in a peripheral hospital.

The MC technique, in which the rectus muscle is only split, causes less postoperative pain than when the muscle is cut; however, it has the disadvantage of limited surgical exposure in obese patients $(5,6)$. In this study, most of the patients were obese, thereby requiring the muscle cutting technique in 37 patients.

The incision length used in MC has been a topic of debate. Some studies have applied the term microlaparotomy for incision $<4 \mathrm{~cm}$, modern mini-laparotomy for 4 $6 \mathrm{~cm}$-incision, conventional minilaparotomy for $6.1-10 \mathrm{~cm}$ incision, and conventional open cholecystectomy for incision longer than $10 \mathrm{~cm}$ (7). The incision length in this study varied from $4.5 \mathrm{~cm}$ to $6 \mathrm{~cm}$. Only routine Deaver's retractors and routine operating room lights were used to perform MC in this study. This is while previous studies have used specially designed retractors such as the Jako retractor system, the stabilized ring retractor, and headlights for performing MC (8-10). 


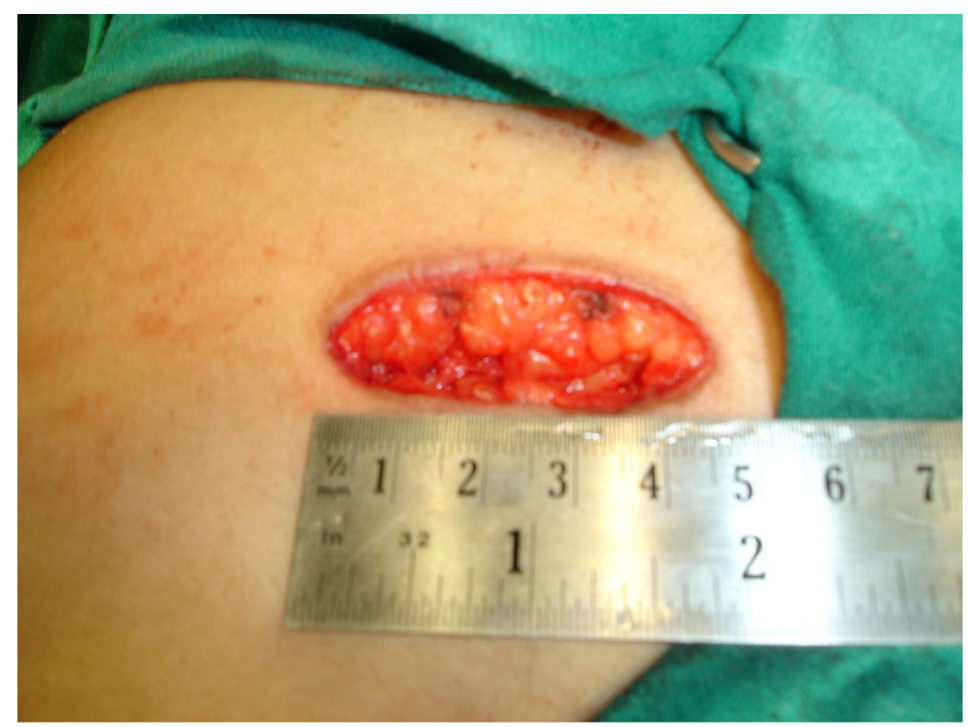

Figure 1. Right subcostal incision $(4.5 \mathrm{~cm})$ for minilaparotomy cholecystectomy

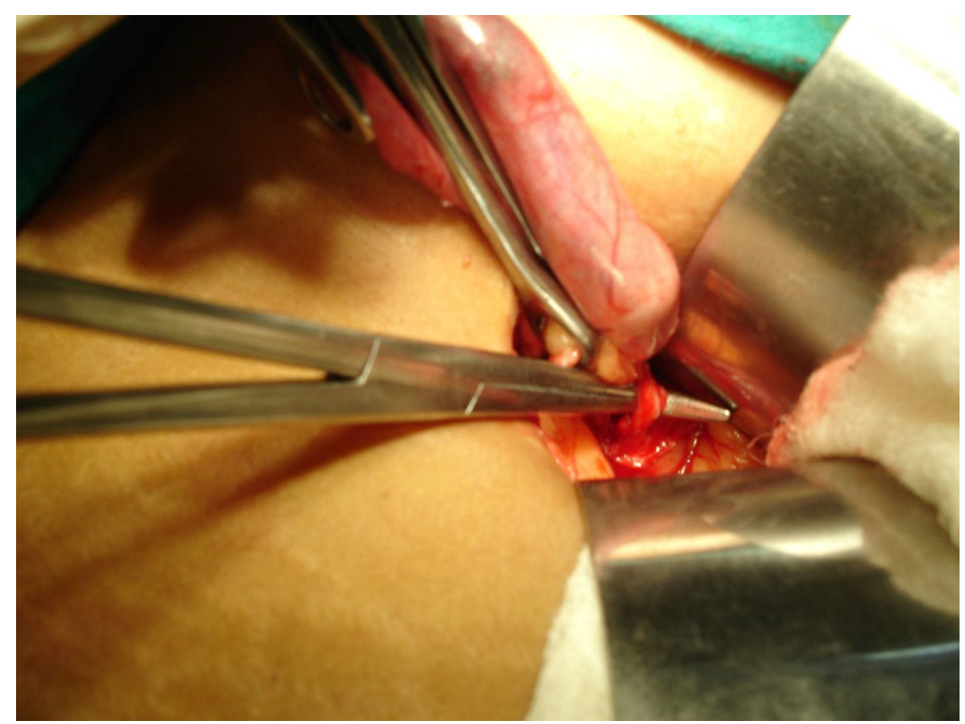

Figure 2. Demonstration of the cystic duct after dissection using 'fundus-first' technique with routine instruments

MC was successfully completed in $94 \%$ of patients, comparable with existing literature $(11,12)$. All the three patients who required the extension of incision had chronically inflamed and severely contracted GB with dense adhesions and difficult dissection. GB was not easily visualized in all these cases, and it had to be identified by palpating the stones in GB. In this study, GB was opened in all these cases, and the stones had to be removed so that GB could be appropriately held for further dissection. The average op- erating time was 60 minutes; it was twice longer (average 125 minutes) in the three patients who required the incision extension, whereas it was 53 minutes in the 47 patients undergoing MC successfully.

The present study revealed that the successful completion of MC using the 'fundus-first' technique is less challenging, which is probably due to the better maneuverability of GB obtained by this technique within the limited surgical exposure of MC. Although this observation has not 


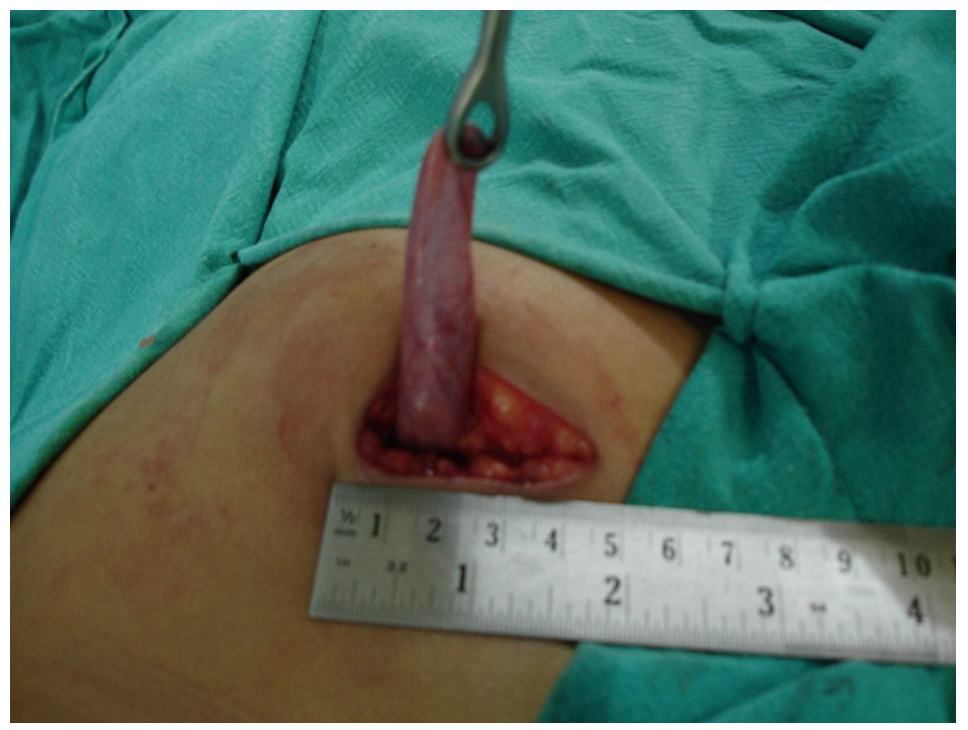

Figure 3. Gall bladder dissected out of a $4.5 \mathrm{~cm}$ right subcostal incision

been documented in the existing literature, some studies have routinely adopted only the 'fundus-first' technique for $\operatorname{MC}(13,14)$.

The incision length is directly proportional to patients' recovery during the postoperative period (15). The average postoperative hospital stay in the present study was 2.14 days (range 2 - 5 days), which is comparable with LC and is much shorter than open cholecystectomy (16). The postoperative hospital stay was longer in the three patients who required incision extension. On the other hand, all other patients were discharged on the second postoperative day. The analgesic requirement in patients with MC is also less compared to conventional open cholecystectomy due to less tissue trauma (15). In this study, most patients in required only oral analgesics after the initial 24 hours of operation. The average time required to return to routine work was nine days (range 7 - 12 days), which is much shorter than that the period observed in conventional open cholecystectomy $(17,18)$.

There was an intra-operative complication in the form of inadvertent small bowel injury in only one patient, which was caused due to difficult anatomy. The same patient had postoperative complications such as wound seroma and later superficial wound infection. There was no mortality in the study, and the patients were satisfied with the cosmetic outcome of the operation. Moreover, there was no long-term morbidity during the follow-up pe- riod (Figure 4).

\subsection{Conclusions}

MC was successfully completed in $94 \%$ of the participants using routine instruments, and it was accompanied with negligible morbidity, no mortality, and satisfactory cosmetic outcome for the patients. Our study observed that it is easier to complete MC using the antegrade or 'fundus-first' approach. MC is undoubtedly better than conventional open cholecystectomy and comparable with LC in terms of all operative parameters. Itis also ideal for peripheral hospitals in developing countries having insufficient laparoscopic facilities.

\section{Footnotes}

Authors' Contribution: Performed surgery for all the patients: Bharath N. Kumar; Study concept and design; Analysis and interpretation of data: Bharath N. Kumar; Drafting and critical revision of the manuscript for important intellectual content: Rahul Pandey.

Conflict of Interests: There is no conflict of interests.

Ethical Approval: This study was approved by the Institutional Ethics committee.

Funding/Support: There was no funding/support.

Informed Consent: Written informed consent was obtained from all patients. 


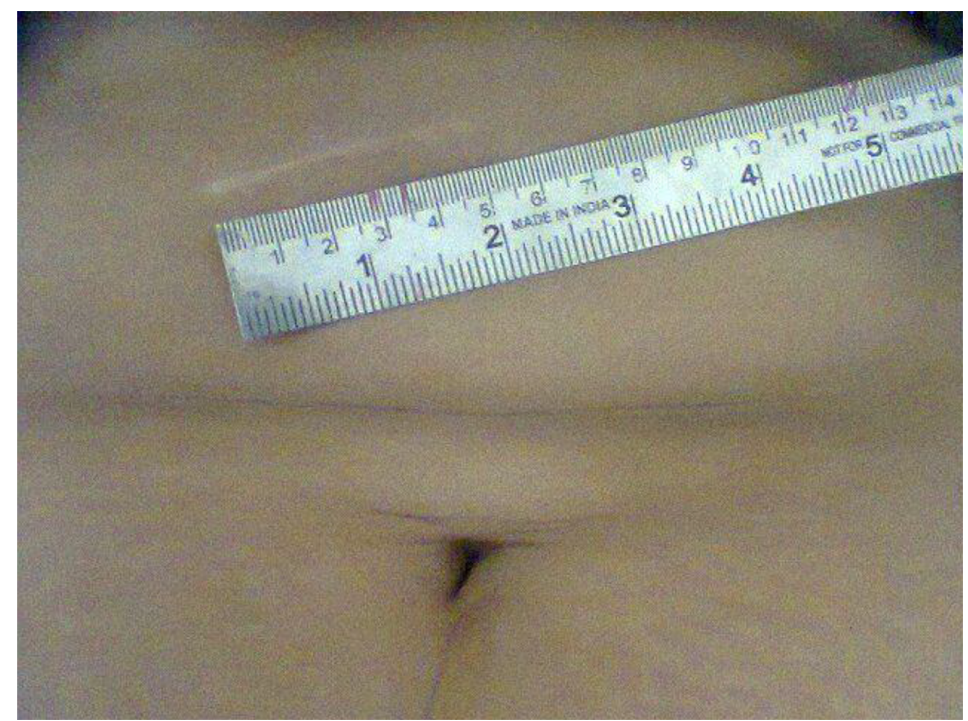

Figure 4. Postoperative scar three months after minilaparotomy cholecystectomy

\section{References}

1. Dubois F, Berthelot B. Cholecystectomy par mini laparotomy. Nouv Presse Med.1982;11(15):1139-41.

2. Rozsos I, Jako G. Microlaparotomy Cholecystectomy. Amy Surg. 1995;222:762-3.

3. Reynolds Jr W. The first laparoscopic cholecystectomy. J Soc Laparoend Surg. 2001;5(1):89-94.

4. Teerawattananon Y, Mugford M. Is it worth offering a routine laparoscopic cholecystectomy in developing countries? A Thailand case study. Cost Eff Resour Alloc. 2005;3:10. doi: 10.1186/1478-7547-3-10. [PubMed: 16259625]. [PubMed Central: PMC1291381].

5. McMahon AJ, Ross S, Baxter JN, Russell IT, Anderson JR, Morran CG, et al. Symptomatic outcome 1 year after laparoscopic and minilaparotomy cholecystectomy: a randomized trial. BrJ Surg. 1995;82(10):137882. doi: 10.1002/bjs.1800821028. [PubMed: 7489171].

6. Shara FG, Ali AH, Bassiony FA, Mareis MM, Smith LM. Minilaparotomy versus laparoscopic cholecystectomy. New Egypt J Med. 1993;9:269-73.

7. Rozsos I, Ferenczy J, Afshin D, Rozsos T. Cholecystectomy performed by macro-and modern mini-laparotomy. Orvosi hetilap. 1995;136(9):475-81.

8. Bhagabati IN. Instruments for minicholecystectomy. Medifaci. 1995;16:15-7.

9. Froschle GW, Kiraly Z, Broelsch CE. [Cholecystectomy by minilaparotomy with the Jako retractor system]. Langenbecks Arch Chir. 1997;382(5):274-6. doi: 10.1007/BF02395732. [PubMed: 9411175].

10. Russell RC, Shankar S. The stabilized ring retractor: a technique for cholecystectomy. $B r \quad J$ Surg. 1987;74(9):826. doi: 10.1002/bjs.1800740925. [PubMed: 3664250].

11. Daou R. Cholecistectomy using a minilaparotomy Ann. Chir. 1998;52(7):625-8.
12. al-Tameem MM. Minilaparotomy cholecystectomy. J R Coll Surg Edinb. 1993;38(3):154-7. [PubMed: 7687677].

13. Harju J, Paakkonen M, Eskelinen M. Minilaparotomy cholecystectomy as a day surgery procedure: a prospective clinical pilot study. Scand J Surg. 2007;96(3):206-8. doi: 10.1177/145749690709600304. [PubMed: 17966745].

14. Leo J, Filipovic G, Krementsova J, Norblad R, Soderholm M, Nilsson E. Open cholecystectomy for all patients in the era of laparoscopic surgery - a prospective cohort study. BMC Surg. 2006;6:5. doi: 10.1186/1471-2482-6-5. [PubMed: 16584556]. [PubMed Central: PMC1450318].

15. O’Dwyer PJ, McGregor JR, McDermott EW, Murphy JJ, O’Higgins NJ. Patient recovery following cholecystectomy through a $6 \mathrm{~cm}$ or 15 $\mathrm{cm}$ transverse subcostal incision: a prospective randomized clinical trial. Postgrad Med J.1992;68(804):817-9. doi: 10.1136/pgmj.68.804.817. [PubMed: 1461854]. [PubMed Central: PMC2399524].

16. Keus F, Gooszen HG, van Laarhoven CJ. Open, small-incision, or laparoscopic cholecystectomy for patients with symptomatic cholecystolithiasis. An overview of Cochrane Hepato-Biliary Group reviews. Cochrane Database Syst Rev. 2010;(1). CD008318. doi: 10.1002/14651858.CD008318. [PubMed: 20091665]. [PubMed Central: PMC7180153].

17. Assalia A, Schein M, Kopelman D, Hashmonai M. Minicholecystectomy vs conventional cholecystectomy: a prospective randomized trial-implications in the laparoscopic era. World J Surg.1993;17(6):7559. doi: 10.1007/BF01659087. [PubMed: 8109113].

18. Chalkoo M, Ahangar S, Durrani AM, Chalkoo S, Shah MJ, Bashir MI. Mini-lap cholecystectomy: modifications and innovations in technique. Int J Surg. 2010;8(2):112-7. doi: 10.1016/j.ijsu.2009.11.007. [PubMed: 19944194]. 